\title{
Nonlinear design model of reinforced concrete in terms of triaxial deformation
}

\author{
Mikhail Berlinov ${ }^{1}$ and Artem Grigorjan ${ }^{1}$ \\ ${ }^{1}$ Moscow State University of Civil Engineering, Yaroslavskoe shosse, 26, Moscow, 129337, Russia
}

\begin{abstract}
The influence of reinforcement in the concrete work in the conditions of triaxial stress state high-rise construction is considered. The basic phenomenological resolving equations in a triaxial stress-strain state under vibration effects were developed for the calculation of reinforced concrete elements working with and without cracks in terms of nonlinearity and rheology of concrete deformation. A method for linearizing a problem based on integral estimates is proposed, which provides for the fixation of the creep processes in the considered period of time. The implementation of this method will allow us to find a numerical solution of the problem. Such an approach in the design of reinforced concrete structures will allow not only more fully taking into account the real conditions of their work, revealing additional reserves of load capacity, but also opening additional opportunities for analysis and forecasting their functioning at various stages of operation.
\end{abstract}

\section{Introduction}

In the case of a real deformation to take into account the work of reinforcement, and consideration should be implemented for the two cases to the appearance of cracks in the concrete and after their appearance [1].

All existing concrete deformation models in the absence of cracking accepted the hypothesis of simultaneous linear deformation of reinforcement and concrete, which makes it possible simply to take into account the effect of reinforcement on the rigidity of reinforced concrete under axial and shear deformation, the effect of reinforcement on the rigidity of the elements in a direction transverse to the direction of the rods, the impact more obliquely installed reinforcement, as well as - the weakening of the concrete reinforcement channels.

The adoption of such assumptions can create different calculation methods, by introducing a model of various factors to obtain various physical equations, significantly simplifying the calculation [2].

In general, the volume of orthotropic reinforcement "flows" rods coincide with the directions of the coordinate axes $\mathrm{x}, \mathrm{y}, \mathrm{z}$, (Fig.1). The rods in voluminous carcasses are arranged with a certain pitch $\mathrm{a}_{\mathrm{x}}, \mathrm{a}_{\mathrm{y}}, \mathrm{a}_{\mathrm{z}}$, along the respective axes, and each area is characterized by an appropriate factor reinforcing $\mu_{\mathrm{si}}(\mathrm{i}=\mathrm{x}, \mathrm{y}, \mathrm{z})$ is the area of rods per unit area normally located to the respective direction of the rods:

\footnotetext{
* Corresponding author: berlinov2010@mail.ru
} 


$$
\mu_{\mathrm{sx}}=\mathrm{A}_{\mathrm{sx}} / \mathrm{a}_{\mathrm{y}} \mathrm{a}_{\mathrm{z}} ; \quad \mu_{\mathrm{sy}}=\mathrm{A}_{\mathrm{sy}} / \mathrm{a}_{\mathrm{x}} \mathrm{a}_{\mathrm{z}} ; \quad \mu_{\mathrm{sz}}=\mathrm{A}_{\mathrm{sz}} / \mathrm{a}_{\mathrm{x}} \mathrm{a}_{\mathrm{y}} .
$$

The proposed method is, in principle, allows to consider any direction of reinforcement, such as an inclined $\mathrm{A}_{\mathrm{j}}$. In this case, the reinforcement ratio:

$$
\mu_{\mathrm{sj}}=\mathrm{A}_{\mathrm{sj}} / \mathrm{a}_{1} \mathrm{a}_{2}
$$

where: $\mathrm{a}_{1}$ and $\mathrm{a}_{2}$ - the distance between the individual rods with oblique reinforcement.

Inclined rods additionally be characterized by the direction cosines $1_{i j}$ to the directions of the axes $x, y, z$, the values of which are given in [3], and that must be considered when calculating the elements with such reinforcement. Due to the fact that the orthotropic reinforcement is more frequent it is regarded as a primary [4].

It is known that the force in the cross section of an elementary volume of reinforced concrete element prior to the appearance of cracks is the sum of forces perceived concrete and reinforcement. For example along the $\mathrm{x}$ axis (Fig.1), this dependence can be written simply:

$$
N_{x}(t)=N_{b x}(t)+N_{s x}(t)
$$

where: $N_{x}(t)$ - the average force in concrete element; $N_{b x}(t)$ and $N_{s x}(t)$ - respectively forces in concrete and reinforcement.

Turning to stress, we get the following formula:

$$
\sigma_{x}(t) A_{x}=\sigma_{b x}(t) A_{b x}+\sigma_{s x}(t) A_{s x}
$$

here: similar $\sigma_{\mathrm{x}}(\mathrm{t})$ - mean stress in the cross section of the elementary volume concrete element; $\sigma_{b x}(t)$ and $\sigma_{s x}(t)$ - mean stresses in the concrete and reinforcement; $\mathrm{A}_{b x}$ and $\mathrm{A}_{\mathrm{sx}}$ accordingly the average area of concrete and reinforcement in the concrete section.

Assuming that this expression $A_{b x}=A_{x}+A_{s x}$, a reinforcement ratio is the ratio of the reinforcement area to cross-sectional area of the element $\mu_{\mathrm{sx}}=\mathrm{A}_{\mathrm{sx}} / \mathrm{A}_{\mathrm{x}}$, can be easily obtained:

$$
\sigma_{x}(t)=\sigma_{b x}(t) \frac{A_{x}-A_{s x}}{A_{x}}+\sigma_{s x}(t) \frac{A_{s x}}{A_{x}} \quad \text { or : } \quad \sigma_{x}(t)=\left(1-\mu_{s x}\right) \sigma_{b x}(t)+\mu_{s x} \sigma_{s x}(t) .
$$

In equation (5), the first term $\left(1-\mu_{\mathrm{sx}}\right) \sigma_{\mathrm{bx}}(\mathrm{t})$ will be called, in accordance with established traditions, given the stresses in the concrete and $-\mu_{\mathrm{sx}} \sigma_{\mathrm{sx}}(\mathrm{t})$ - given the stresses in the reinforcement.

When used according to (5) should be borne in mind that it is valid only if the area of reinforcement in cross-section comparable to the concrete area, ie when considered sufficiently small volume elements [5]. Otherwise, when the $\mathrm{A}_{\mathrm{sx}} \backslash \mathrm{A}_{\mathrm{x}}$, i.e. It can be taken $A_{x} \cong A_{b x}$ and then the dependence (5) is substantially simplified and becomes:

$$
\sigma_{x}(t)=\sigma_{b x}(t)+\mu_{s x} \sigma_{s x}(t) .
$$

\section{Results section}

In triaxial deformation, determination of stress or strain along one axis, one should consider the impact on these strains and stresses acting along the other two remaining axes. Fig. 2 shows the action of normal and tangential stresses scheme in the volume element of reinforced concrete element, on the basis of this scheme, you can easily get physical strain relations with orthotropic reinforcement. These equation allow us to establish the relationship between reduced stress and strains in components of reinforced concrete section (concrete and reinforcement). 
From the joint consideration of the expressions (5) and (6) you can go to the equations of triaxial deformation and to determine the relationship between the average stresses in the unit cell volume deformation and reduced stress, acting in its components:

$$
\begin{aligned}
& \sigma_{x}(t)=\sigma_{b x}(t) S_{b}\left[\sigma_{b x}(t)\right]-v_{b}\left\{\sigma_{b y}(t) S_{b}\left[\sigma_{b y}(t)\right]+\sigma_{b z}(t) S_{b}\left[\sigma_{b z}(t)\right]\right\}+ \\
& +\mu_{s x} \sigma_{s x}(t) S_{s}\left[\sigma_{s x}(t)\right]+v_{s}\left\{\mu_{s y} \sigma_{s y}(t) S_{s}\left[\sigma_{s y}(t)\right]+\mu_{s z} \sigma_{s z}(t) S_{s}\left[\sigma_{s z}(t)\right]\right\}
\end{aligned}
$$

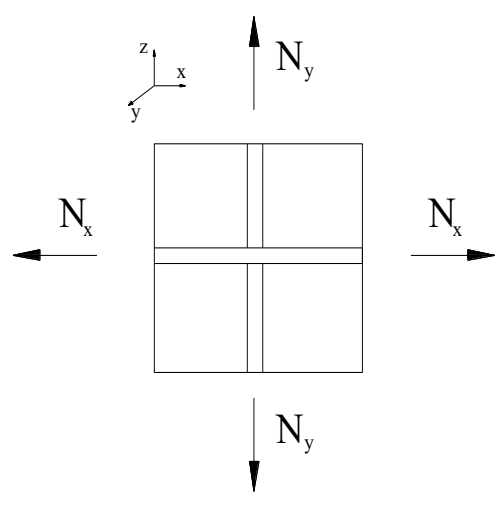

Fig.1. Current forces.

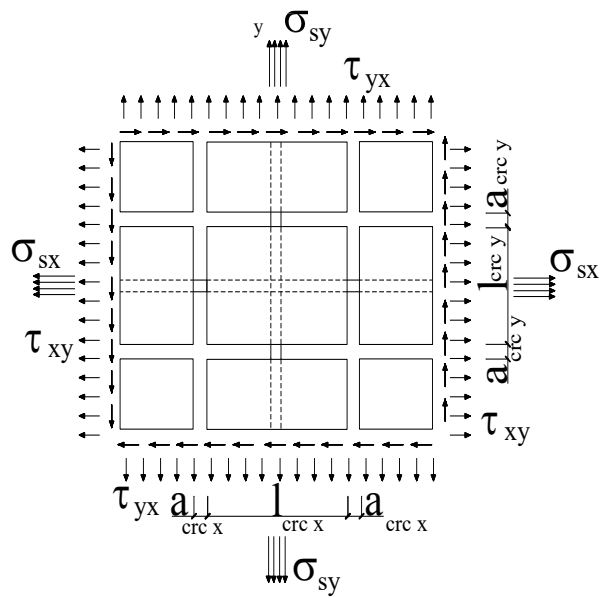

Fig.2. Cracking.

or in the general form:

$$
\begin{aligned}
& \sigma_{x}(t)=\left(1-\mu_{s x}\right) \sigma_{b x}(t) S_{b}\left[\sigma_{b x}(t)\right]-v_{b}\left\{\left(1-\mu_{s y}\right) \sigma_{b y}(t) S_{b}\left\lfloor\sigma_{b y}(t)\right]+\left(1-\mu_{s z}\right) \sigma_{b z}(t) S_{b}\left[\sigma_{b z}(t)\right]\right\}+ \\
& +\mu_{s x} \sigma_{s x}(t) S_{s}\left[\sigma_{s x}(t)\right]+v_{s}\left\{\mu_{s y} \sigma_{s y}(t) S_{s}\left[\sigma_{s y}(t)\right]+\mu_{s z} \sigma_{s z}(t) S_{s}\left[\sigma_{s z}(t)\right]\right\}
\end{aligned}
$$

here: $v_{\mathrm{b}}$ and $v_{\mathrm{s}}$ - respectively, the relative ratios of the transverse strain of concrete and reinforcement; $S_{b}$ and $S_{s}$ - nonlinearity function and deformation of concrete reinforcement; $\mu_{\mathrm{si}}$ - reinforcing factors along the respective axes, defined by formulas (1).

Dependencies (7) and (8), taking into account the accepted hypothesis about the division of strain in the elastic-instantaneous and creep deformation and the mathematical rule on the representation of the definite integral can be interpreted as follows:

$$
\begin{gathered}
\sigma_{x}(t)=\left(1-\mu_{s x}\right) \sigma_{b x m}(t) S_{m}\left[\sigma_{b x m}(t)\right]-v_{b}\left\{\left(1-\mu_{s y}\right) \sigma_{b y m}(t) S_{m}\left[\sigma_{b y m}(t)\right]+\left(1-\mu_{s z}\right) \sigma_{b z m}(t) S_{m}\left[\sigma_{b z m}(t)\right]\right\}- \\
-\int_{t_{0}}^{t} \frac{\partial}{\partial \tau}\left(1-\mu_{s x}\right) \sigma_{b x p}(t) S_{p}\left[\sigma_{b x p}(t)\right] d \tau+v_{b} \int_{t_{0}}^{t} \frac{\partial}{\partial \tau}\left\{\left(1-\mu_{s y}\right) \sigma_{b y p}(t) S_{p}\left[\sigma_{b y p}(t)\right]+\left(1-\mu_{s z}\right) \sigma_{b s z}(t) S_{p}\left[\sigma_{b z p}(t)\right]\right\} d \tau+ \\
+\mu_{s x} \sigma_{s x}(t) S_{s}\left[\sigma_{s x}(t)\right]+v_{s}\left\{\mu_{s y} \sigma_{s y}(t) S_{s}\left[\sigma_{s y}(t)\right]+\mu_{s z}(t) S_{s}\left[\sigma_{s z}(t)\right]\right\}
\end{gathered}
$$

Based on the known compatibility condition of deformation of concrete and reinforcement in areas of reinforced concrete structures operating without cracks, i.e., considering that:

$$
\varepsilon_{b x}(t)=\varepsilon_{s x}(t)
$$

where: $\varepsilon_{b x}(t)$ and $\varepsilon_{s x}(t)$, respectively, and deformation of concrete reinforcement along the $\mathrm{x}$-axis at a given moment of time. 
Considering the deformation of concrete taking into account the already mentioned hypothesis about the division of strains can be obtained:

$$
\varepsilon_{b x}(t)=\varepsilon_{b x m}(t)+\varepsilon_{b x p}(t)-v_{b}\left[\varepsilon_{b y m}(t)+\varepsilon_{b y p}(t)+\varepsilon_{b z m}(t)+\varepsilon_{b z p}(t)\right] ;
$$

here $\varepsilon_{\text {bim }}(\mathrm{t})$ and $\varepsilon_{\text {bip }}(\mathrm{t})$ - respectively elastically instantaneous deformation and creep.

Turning to stress can be written the following relationship:

$$
\begin{aligned}
& \varepsilon_{b x}(t)= \frac{\sigma_{b x m}(t)}{E_{m}^{0}(t)} S_{m}\left[\sigma_{b m x}(t)\right]-v_{b}\left\{\frac{\sigma_{b y y}(t)}{E_{m}^{0}(t)} S_{m}\left[\sigma_{b y m}(t)\right]+\frac{\sigma_{b z z}(t)}{E_{m}^{0}(t)} S_{m}\left[\sigma_{b m z}(t)\right]\right\}+\sigma_{b x p}(t) S_{p}\left[\sigma_{b x p}(t)\right] K\left(\sigma_{b x p}, \omega_{\min }, \omega, t\right) C\left(t, t_{0}\right)- \\
&-v_{b}\left\{\sigma_{b y p}(t) S_{p}\left[\sigma_{b y p}(t)\right] K\left(\sigma_{b y p}, \omega_{\min }, \omega, t\right)+\sigma_{b z p}(t) S_{p}\left[\sigma_{b z p}(t)\right]\right\} K\left(\sigma_{b z p}, \omega_{\min }, \omega, t\right) C\left(t, t_{0}\right)- \\
& \quad-\int_{t_{0}}^{t}\left\langle\sigma_{b x p}(\tau) S_{p}\left[\sigma_{b x p}(\tau)\right] K\left(\sigma_{b x p}, \omega_{\min }, \omega, \tau\right)-v_{b}\left\{\sigma_{b y p}(\tau) S_{p}\left[\sigma_{b y p}(\tau)\right] K\left(\sigma_{b y p}, \omega_{\min }, \omega, t\right)-\right.\right. \\
&\left.\left.-\sigma_{b z p}(\tau) S_{p}\left[\sigma_{b z p}(\tau)\right] K\left(\sigma_{b z p}, \omega_{\min }, \omega, \tau\right)\right\}\right\} \frac{\partial}{\partial \tau} C(\tau, t) d \tau
\end{aligned}
$$

where: $\mathrm{C}\left(\mathrm{t}, \mathrm{t}_{0}\right)$ and $\mathrm{K}\left(\sigma, \omega_{\min }, \omega, \mathrm{t}\right)$ are the creep measure and the vibration creep factor.

Considering the deformation of the reinforcing steel, with similar positions, we have:

$$
\begin{aligned}
& \varepsilon_{s x}(t)=\frac{\sigma_{s x m}(t)}{E_{s}^{0}(t)} S_{m}\left[\sigma_{s m x}(t)\right]-v_{b}\left\{\frac{\sigma_{s y m}(t)}{E_{s}^{0}(t)} S_{m}\left[\sigma_{s y m}(t)\right]+\frac{\sigma_{s z m}(t)}{E_{s}^{0}(t)} S_{m}\left[\sigma_{s m z}(t)\right]\right\} \\
& +\sigma_{s x p}(t) S_{p}\left[\sigma_{s p x}(t)\right] C\left(t, t_{0}\right)-v_{s}\left\{\sigma_{s y p}(t) S_{p}\left[\sigma_{s y p}(t)\right]+\sigma_{s z p}(t) S_{p}\left[\sigma_{s z p}(t)\right]\right\} C\left(t, t_{0}\right)- \\
& -\int_{t_{0}}^{t}\left\langle\sigma_{s x p}(\tau) S_{p}\left[\sigma_{s x p}(\tau)\right] K\left(\sigma_{s x p}, \omega_{\min }, \omega, \tau\right)-v_{b}\left\{\sigma_{s y p}(\tau) S_{p}\left[\sigma_{s y p}(\tau)\right] K\left(\sigma_{s y p}, \omega_{\min }, \omega, \tau\right)-\right.\right. \\
& \left.\left.-\sigma_{s z p}(\tau) S_{p}\left[\sigma_{s z p}(\tau)\right] K\left(\sigma_{s z p}, \omega_{\min }, \omega, \tau\right)\right\}\right\rangle \frac{\partial}{\partial \tau} C_{s}(\tau, t) d \tau
\end{aligned}
$$

in the formulas (13), the index s means that it is a reinforcement strains.

Comparing equations (12) and (13) based on the identity (10) we can receive the relationship for the unknown desired values for which are received in the armature stresses. Solving this equation using a consistent comparison of longitudinal and transverse strains, weobtain:

$$
\begin{aligned}
& \sigma_{s x}=\sigma_{b x m}(t) \frac{E_{s}}{E_{m}^{0}(t)} S_{m}\left[\sigma_{b x m}(t)\right]-\frac{v_{b}}{v_{s}} \frac{E_{s}}{E_{m}^{0}(t)}\left\{\sigma_{b y m} S_{m}\left[\sigma_{b y m}(t)\right]+\sigma_{b z m}(t) S_{m}\left[\sigma_{b z m}(t)\right]\right\}+ \\
& +\sigma_{b x p}(t) S_{p}\left[\sigma_{b x p}(t)\right] \frac{K\left(\sigma_{b x p}, \omega_{\min }, \omega, t\right) C\left(t, t_{0}\right)}{K\left(\sigma_{s x p}, \omega_{\min }, \omega, t\right) C_{s}\left(t, t_{0}\right)}-\frac{v_{b}}{v_{s}}\left\{\begin{array}{l}
\sigma_{b y p}(t) S_{p}\left[\sigma_{b y p}(t)\right] \frac{K\left(\sigma_{b y p}, \omega_{\min }, \omega, t\right)}{K\left(\sigma_{s y p}, \omega_{\min } \omega, t\right)}+ \\
+\sigma_{b z p}(t) S_{p}\left[\sigma_{b z p}(t)\right] \frac{K\left(\sigma_{b z p}, \omega_{\min }, \omega, t\right)}{K\left(\sigma_{s z p}, \omega_{\min }, \omega, t\right)}
\end{array}\right\} . \\
& \cdot \frac{C\left(t, t_{0}\right)}{C_{s}\left(t, t_{0}\right)}-\int_{t_{0}}^{t} \frac{\partial}{\partial \tau} \sigma_{s x p}(\tau) S_{p}\left[\sigma_{b x p}(\tau)\right] \frac{K\left(\sigma_{b x p}, \omega_{\min }, \omega, \tau\right) C(t, \tau)}{K_{s}\left(\sigma_{s x p}, \omega_{\min }, \omega, \tau\right) C_{s}(t, \tau)} \\
& +\frac{v_{b}}{v_{s}} \int_{t_{0}}^{t} \frac{\partial}{\partial \tau}\left\{\sigma_{b y p}(\tau) S_{p}\left[\sigma_{b y p}\right](\tau) \frac{K\left(\sigma_{b y p}, \omega_{\min }, \omega, \tau\right)}{K_{s}\left(\sigma_{s y p}, \omega_{\min } \omega, \tau\right)}+\sigma_{b z p} S_{p}\left[\sigma_{b z p}(\tau)\right] \frac{K\left(\sigma_{b z p}, \omega_{\min }, \omega, \tau\right)}{K_{s}\left(\sigma_{s z p}, \omega_{\min }, \omega, \tau\right)}\right\} \frac{C(t, \tau)}{C_{s}(t, \tau)} d \tau
\end{aligned}
$$

Substituting this value in (9) on the basis of (10) and taking into account that $\alpha=\mathrm{E}_{\mathrm{s}} / \mathrm{E}_{\mathrm{m}}^{0}(\mathrm{t})$ - the well-known relation modules of elastic deformation of the reinforcing steel and concrete, called the coefficient of reduction reinforcement to concrete can be obtained values of average stresses based the impact of steel reinforcement along the $\mathrm{x}$ axis. If we make a similar argument, it is easy enough to get, and the other two components of the normal stresses on the two remaining axes y and $z$. These components will not lead by virtue of their 
large bulkiness they just get on the basis of the formula below using a circular permutation of indices in stress and reinforcement ratios (15).

For some types of reinforcing steel working as part of reinforced concrete structures, which include high-strength steel, and certain classes of thermally bonded reinforcement, nonlinear deformation and rheology of which is extremely small, in order to simplify settlement of dependencies this factor can be ignored. In addition, for the same purpose can be extended this assumption and other types of reinforcing steel due to the following reasons: under normal conditions of use, stresses in the reinforcement of concrete structures operating without cracks substantially less than the maximum allowable; secondly, in the reinforcing steel and the creep phenomenon vibrocreep in standard conditions and slightly manifested at sufficiently high temperatures, where normal use of reinforced concrete structures are not intended.

$$
\begin{aligned}
& \sigma_{x}=\left(1-\mu_{s x}\right) \sigma_{b x m}(t) S_{m}\left[\sigma_{b x m}(t)\right]\left(1+\alpha \mu_{s x}\right)-v_{b}\left\{\begin{array}{l}
\left(1-\mu_{s y}\right) \sigma_{b y m} S_{m}\left[\sigma_{b y m}(t)\right]\left(1+\alpha \mu_{s y}\right)+ \\
+\left(1-\mu_{s z}\right) \sigma_{b z m}(t) S_{m}\left[\sigma_{b z m}(t)\right]\left(1+\alpha \mu_{s z}\right)
\end{array}\right\}+ \\
& \left.+\left(1-\mu_{s x}\right) \sigma_{b x p}(t) S_{p}\left[\sigma_{b x p}(t)\right]\left[1+\mu_{s x} \frac{K\left(\sigma_{b x p}, \omega_{\min }, \omega, t\right)}{K_{s}\left(\sigma_{s x p}, \omega_{\min }, \omega, t\right)} \frac{C(t, \tau)}{C_{s}(t, \tau)}\right]\right] C\left(t, t_{0}\right)- \\
& -v_{b}\left\{\begin{array}{l}
\left.\left(1-\mu_{s y}\right) \sigma_{b y p}(t) S_{p}\left[\sigma_{b y p}(t)\right]\left[1+\mu_{s y} \frac{K\left(\sigma_{b y p}, \omega_{\min }, \omega, t\right)}{K_{s}\left(\sigma_{s y p}, \omega_{\min } \omega, t\right)} \frac{C(t, \tau)}{C_{s}(t, \tau)}\right]\right]+ \\
\left.+\left(1-\mu_{s z}\right) \sigma_{b z p}(t) S_{p}\left[\sigma_{b z p}(t)\right] 1+\mu_{s z} \frac{K\left(\sigma_{b z p}, \omega_{\min }, \omega, t\right)}{K_{s}\left(\sigma_{s z p}, \omega_{\min }, \omega, t\right)} \frac{C(t, \tau)}{C_{s}(t, \tau)}\right]
\end{array}\right\} C\left(t . t_{0}\right)+ \\
& +\int_{t_{0}}^{t}\left(1-\mu_{s y}\right) \sigma_{b x p}(\tau) S_{p}\left[\sigma_{b x p}(\tau)\right]\left[1+\mu_{s x} \frac{K\left(\sigma_{b x p}, \omega_{\min }, \omega, \tau\right)}{K_{s}\left(\sigma_{s x p}, \omega_{\min }, \omega, \tau\right)} \frac{C(t, \tau)}{C_{s}(t, \tau)}\right] \frac{\partial}{\partial \tau} C(t, \tau)+ \\
& +v_{b} \int_{t_{0}}^{t}\left\{\begin{array}{l}
\left.\left(1-\mu_{s y}\right) \sigma_{b y p}(\tau) S_{p}\left[\sigma_{b y p}(\tau)\right]\left[1+\mu_{s y} \frac{K\left(\sigma_{b y p}, \omega_{\min }, \omega, t\right) C(t, \tau)}{K_{s}\left(\sigma_{s y p}, \omega_{\min }, \omega, t\right) C_{s}(t, \tau)}\right]+\right] \\
+\left(1-\mu_{s z}\right) \sigma_{b z p}(\tau) S_{p}\left[\sigma_{b z p}(\tau)\right]\left[1+\mu_{s z} \frac{K\left(\sigma_{b z p}, \omega_{\min }, \omega, t\right) C(t, \tau)}{K_{s}\left(\sigma_{s z p}, \omega_{\min }, \omega, t\right) C_{s}(t, \tau)}\right]
\end{array}\right\} \frac{\partial}{\partial \tau} C(t, \tau)
\end{aligned}
$$

In this case, deformation of the steel reinforcement is possible (but not necessarily) be considered independent of the nonlinearity of the deformation and the time factor and be taken into account in a certain elastic-linear form:

$$
\varepsilon_{s x}=\frac{1}{E_{s}}\left[\sigma_{s x}-v_{s}\left(\sigma_{s y}+\sigma_{s z}\right)\right] \quad \varepsilon_{s y}=\frac{1}{E_{s}}\left[\sigma_{s y}-v_{s}\left(\sigma_{s x}+\sigma_{s z}\right)\right] \quad \varepsilon_{s z}=\frac{1}{E_{s}}\left[\sigma_{s z}-v_{s}\left(\sigma_{s x}+\sigma_{s y}\right)\right]
$$

Then the formula (14) in the case of cancellation of nonlinearity and nonequilibrium deformation reinforcement substantially simplified and takes the following form:

$$
\begin{aligned}
& \sigma_{s x}=\sigma_{b x m}(t) \frac{E_{s}}{E_{m}^{0}(t)} S_{m}\left[\sigma_{b x m}(t)\right]-\frac{v_{b}}{v_{s}} \frac{E_{s}}{E_{m}^{0}(t)}\left\{\sigma_{b y m} S_{m}\left[\sigma_{b y m}(t)\right]+\sigma_{b z m}(t) S_{m}\left[\sigma_{b z m}(t)\right]\right\}-\sigma_{b x p}(t) . \\
& \cdot S_{p}\left[\sigma_{b x p}(t)\right] E_{s} K\left(\sigma_{b x p}, \omega_{\min }, \omega, t\right) C\left(t, t_{0}\right)-\frac{v_{b}}{v_{s}}\left\{\begin{array}{c}
\sigma_{b y p}(t) S\left[\sigma_{b y p}(t)\right] K\left(\sigma_{b y p}, \omega_{\min }, \omega, t\right)+ \\
+\sigma_{b z p}(t) S_{p}\left[\sigma_{b z p}(t)\right] K\left(\sigma_{b z p}, \omega_{\min }, \omega, t\right)
\end{array}\right\} C\left(t, t_{0}\right)- \\
& -\int_{t_{0}}^{t} \frac{\partial}{\partial \tau} E_{s} \sigma_{b x p}(\tau) S_{p}\left[\sigma_{b x p}(\tau)\right] K\left(\sigma_{b x p}, \omega_{\min }, \omega, \tau\right) C(t, \tau) d \tau+ \\
& +\frac{v_{b}}{v_{s}} E_{s} \int_{t_{0}}^{t} \frac{\partial}{\partial \tau}\left\{\sigma_{b y p}(\tau) S_{p}\left[\sigma_{b y p}\right](\tau) K\left(\sigma_{b y p}, \omega_{\min }, \omega, \tau\right)+\sigma_{b z p} S_{p}\left[\sigma_{b z p}(\tau)\right] K\left(\sigma_{b z p}, \omega_{\min }, \omega, \tau\right)\right\} C(t, \tau) d \tau
\end{aligned}
$$


Substituting this value in (9) on the basis of (10) and taking into account that $\alpha=\mathrm{Es}_{\mathrm{s}} / \mathrm{E}_{\mathrm{m}}^{0}(\mathrm{t})$ can be obtained values of the average stresses with the influence of the steel reinforcement along the $\mathrm{x}$ axis. Carrying out similar arguments, we get the values and the other two remaining stress components along the axes y and $\mathrm{z}$ :

$$
\left.\begin{array}{l}
\quad \sigma_{x}(t)=\left(1-\mu_{s x}\right) \sigma_{b x m}(t) S_{m}\left[\sigma_{b x m}(t)\right]\left[1+\alpha \mu_{s x}\right]- \\
\quad-v_{b}\left\{\left(1-\mu_{s y}\right) \sigma_{b y m}(t) S_{m}\left[\sigma_{b y m}(t)\left[1+\alpha \mu_{s y}\right]+\left(1-\mu_{s z}\right) \sigma_{b z m}(t) S_{m}\left[\sigma_{b z m}(t)\right]\left[1+\alpha \mu_{s z}\right]\right\}+\right. \\
+\left(1-\mu_{s x}\right) \sigma_{b x p}(t) S_{p}\left[\sigma_{b x p}(t)\right]\left[1+\mu_{s x} E_{s} K\left(\sigma_{b x p}, \omega_{\min }, \omega, t\right)\right] C\left(t, t_{0}\right)-
\end{array}\right\}
$$

Similar arguments can be made in the analysis of shear stresses i.e.:

$$
T_{x y}=T_{b x y}+T_{s x y}
$$

here: $T_{b x y}$ and $T_{\text {sxy }}$ - tangential forces in the concrete and reinforcement, acting on the faces of the elementary volume of reinforced concrete element to the cracks (Fig. 2.), in contrast to the previously considered normal.

$$
\tau_{x y} A_{x y}=\tau_{b x y} A_{b x y}+\tau_{s x y} A_{s x y}
$$

With the reinforcing channels into account, assuming as before $A_{x y}=A_{b x y}+A_{s x y}$ or $A_{b x y}$ $=A_{x y}-A_{s x y}$ :

$$
\tau_{x y}=\tau_{b x y} \frac{A_{x y}-A_{s x y}}{A_{x y}}+\tau_{s x y} \frac{A_{s x y}}{A_{x y}}=\left(1-\mu_{s x y}\right) \tau_{b x y}+\mu_{s x y} \tau_{s x y}
$$

When considering the shear stresses should be used the dependence of reflecting the conditions of compatibility of strains for the shear stresses:

$$
\tau_{b x y}=\tau_{s x y}
$$

Carrying out similar arguments for shear stresses and lowering obvious intermediate mathematical calculations we obtain:

$$
\begin{aligned}
& \left.\left.\tau_{x y}(t)=\left(1-\mu_{s x y}\right) \tau_{b x y m}(t) S_{m} \mid \tau_{b x y m}(t) \| 1+\alpha k_{v} \mu_{s x y}\right\rfloor+\left(1-\mu_{s x y}\right) \tau_{b x y p}(t) S_{p} \mid \tau_{b x x p}(t)\right] \cdot \\
& \quad \cdot\left[1+\mu_{s x y} k_{v} E_{s} K\left(\tau_{b x y p}, \omega_{\min }, \omega, t\right)\right] C\left(t, t_{0}\right)- \\
& -\left(1-\mu_{s x y}\right) \int_{t_{0}}^{t} \tau_{b x y p}(\tau) S_{p}\left[\tau_{b x y p}(\tau)\right]\left[1+\mu_{s x y} E_{s} k_{v} K\left(\tau_{b x x p}, \omega_{\min }, \omega, \tau\right) \frac{\partial}{\partial \tau} C(t, \tau)\right] d \tau
\end{aligned}
$$

here: $\mu_{\mathrm{xy}}, \mu_{\mathrm{yz}}$ and $\mu_{\mathrm{zx}}$ - thus reinforcement ratios in the direction of the respective axes;

in these equations $k_{v}=\left(1+v_{b}\right) /\left(1+v_{s}\right)$ takes into account the difference in the relative transverse strain of concrete and reinforcement.

Assuming, according to the entry for the previously accepted that the relative ratios of the transverse reinforcement and concrete strains are constants respectively equal $v_{\mathrm{s}}=0,3, v_{\mathrm{b}}=$ 
0,2 , the value of the coefficient $\mathrm{k}$ will be a constant equal to $k_{v}=\left(1+v_{b}\right) /\left(1+v_{s}\right)=(1+0,2) /(1+0,3)=0,923 \cong 1$

Note that the law of pairing shear stresses in the case of deformation of reinforced concrete structures is just not always. From Fig. 1 that it takes place only in the absence or presence of reinforcement of the same diameter in all three mutually perpendicular planes, that is a special case. In actual use concrete structures in the construction of the settlement systems of equations finite difference method or the finite element method when considering the entire area of deformation can meet the cell as a different reinforcement in various areas, and lack of it along one or two axes. In this case, law pairing shear stresses will not be performed and the material elementary volume should be treated as orthotropic with different properties in different directions, which considerably complicate the calculation. In order to simplify, and given the insignificant effect of shear stresses on the overall picture of the stress - strain state (except for pure torsion) agree to consider the law of pairing shear stresses fair. In this case it is advisable to average value ratio reinforcement along the respective axes and take them in the following form:

$\mu_{s x y}=\mu_{s y x}=\frac{\mu_{s x}+\mu_{s y}}{2} ; \mu_{s x z}=\mu_{s z x}=\frac{\mu_{s x}+\mu_{s z}}{2} ; \mu_{s y z}=\mu_{s z y}=\frac{\mu_{s y}+\mu_{s z}}{2}$.

Thus, according to (23) reinforcement ratios $\mathrm{s}$ for the computer account taken differentially, depending on the presence or absence of reinforcement along the respective axes. If the reinforcement is completely absent law pairing is considered valid if the reinforcement is available in all areas, the values of the reinforcement ratios received by the formulas (24), if the reinforcement is not in the direction of any axis, the coefficients of the reinforcement taken on the same dependencies, with the only difference that in the absence of reinforcement direction $\mu_{\mathrm{si}}=0$ value.

Substituting the stresses of the formulas (20) and (24) according to (22), we obtain the values of concrete strain under triaxial stress-strain state running without cracks:

$$
\begin{aligned}
& \varepsilon_{x}\left(t, t_{0}\right)=\frac{\left(1-\mu_{s x}\right)\left(1+\alpha \mu_{s x}\right) \sigma_{b x m}(t) S_{m}\left[\sigma_{b x m}(t)\right]-v_{b}\left\{\begin{array}{l}
\left(1-\mu_{s y}\right)\left(1+\alpha \mu_{s y}\right) \sigma_{y}(t) S_{m}\left[\sigma_{b y m}(t)\right]+ \\
+\left(1-\mu_{s z}\right) \sigma_{b z m}(t) S_{m}\left[\sigma_{b z m}(t)\right]
\end{array}\right\}}{E_{m}^{0}(t)}+ \\
& +\left(1-\mu_{s x}\right) \sigma_{b x p}(t) S_{p}\left[\sigma_{b x p}(t)\right]\left[1+\alpha_{\tilde{N}} \mu_{s x}\right] C\left(t, t_{0}\right)- \\
& -v_{b}\left\{\begin{array}{l}
\left(1-\mu_{s y}\right) \sigma_{b y p}(t) S_{p}\left[\sigma_{b y p}(t)\right]\left[1+\alpha_{C} \mu_{s y}\right]+ \\
+\left(1-\mu_{s z}\right) \sigma_{b z p} S_{p}\left[\sigma_{b z p}(t)\right]\left[1+\alpha_{C} \mu_{s z}\right]
\end{array}\right\} C\left(t, t_{0}\right)- \\
& -\left(1-\mu_{s x}\right) \int_{t_{0}}^{t} \sigma_{b x p}(\tau) S_{p}\left[\sigma_{b x p}(\tau)\right]\left[1+\alpha_{C} \mu_{s x} \frac{\partial}{\partial \tau} C(\tau, t) d \tau\right] d \tau+ \\
& +v_{b} \int_{t_{0}}^{t}\left\{\left(1-\mu_{s y}\right) \sigma_{b y p}(\tau) S_{p}\left[\sigma_{b y p}(\tau)\right]\left[1+\alpha_{C} \mu_{s y}\right]+\right. \\
& +\left(1-\mu_{s z}\right) S_{p}\left[\sigma_{b z p}(\tau)\right]\left[1+\alpha_{C} \mu_{s z}\right\} \frac{\partial}{\partial \tau} C(t, \tau) d \tau \\
& \gamma_{x y}=\frac{2(1+v)}{E_{m}^{0}(t)}\left(1-\mu_{s y y}\right) \tau_{b x y m}(t) S_{m}\left[\tau_{b x y m}(\tau)\left[1+\alpha \mu_{s y y}\right]-2(1+v)\left(1-\mu_{s y y}\right) \tau_{b x p p}(t) S_{p}\left[\tau_{b x p p}(t)\right] .\right. \\
& \cdot\left[1+\alpha_{C} \mu_{s y}\right] C\left(t, t_{0}\right)- \\
& -2(1+v)\left(1-\mu_{s y y}\right) \int_{t_{0}}^{t} \tau_{b y p p}(\tau) S_{p}\left[\tau_{b y p p}(\tau)\left[1+\alpha_{C} \mu_{s x y}\right] d \tau\right.
\end{aligned}
$$


Note that in this expression ratio $\alpha_{\mathrm{c}}=\mathrm{K}\left(\sigma_{\mathrm{bp}}, \omega_{\min }, \omega, \tau\right) \mathrm{C}(\mathrm{t}, \tau) / \mathrm{K}_{\mathrm{s}}\left(\sigma_{\mathrm{sp}}, \omega_{\min }, \omega, \tau\right) \mathrm{C}_{\mathrm{s}}(\mathrm{t}, \tau)$ can be considered as reinforcement reduction coefficient for concrete, interpreted with respect to the creep deformation in contrast to the values of $\alpha=E_{s} / E^{0}(t)$ is valid for elastic-instantaneous deformations.

In case of cancellation of accounting of non-linearity and rheology deformation of reinforcing steel, the equation will look like this:

$$
\begin{aligned}
& \varepsilon_{x}\left(t, t_{0}\right)=\frac{\left(1-\mu_{s x}\right)\left(1+\alpha \mu_{s x}\right) \sigma_{b x m}(t) S_{m}\left[\sigma_{b x m}(t)\right]-v_{b}\left\{\begin{array}{l}
\left(1-\mu_{s y}\right)\left(1+\alpha \mu_{s y}\right) \sigma_{y}(t) S_{m}\left[\sigma_{b y m}(t)\right]+ \\
+\left(1-\mu_{s z}\right) \sigma_{b z m}(t) S_{m}\left[\sigma_{b z m}(t)\right]
\end{array}\right\}}{E_{m}^{0}(t)}+ \\
& +\left(1-\mu_{s x}\right) \sigma_{b x p}(t) S_{p}\left[\sigma_{b x p}(t)\right]\left[1+\mu_{s x} E E_{s} K\left(\sigma_{b x p}, \omega_{\min }, \omega, t\right)\right] C\left(t, t_{0}\right)- \\
& -v_{b}\left\{\begin{array}{l}
\left(1-\mu_{s y}\right) \sigma_{b y p}(t) S_{p}\left[\sigma_{b y p}(t)\right]\left[1+\mu_{s y} E_{s} K\left(\sigma_{b y p}, \omega_{\min }, \omega, t\right)\right]+ \\
+\left(1-\mu_{s z}\right) \sigma_{b z p} S_{p}\left[\sigma_{b z p}(t)\right]\left[1+\mu_{s z} E_{s} K\left(\sigma_{b z p}, \omega_{\min }, \omega, t\right)\right]
\end{array}\right\} C\left(t, t_{0}\right)- \\
& -\left(1-\mu_{s x}\right) \int_{t_{0}}^{t} \sigma_{b x p}(\tau) S_{p}\left[\sigma_{b x p}(\tau)\right]\left[1+\mu_{s x} E_{s} K\left(\sigma_{b x p}, \omega_{\min }, \omega, \tau\right) \frac{\partial}{\partial \tau} C(\tau, t) d \tau\right] d \tau+ \\
& +v_{b} \int_{t_{0}}^{t}\left\{\left(1-\mu_{s y}\right) \sigma_{b y p}(\tau) S_{p}\left[\sigma_{b y p}(\tau)\right]\left[1+\mu_{s y} E_{s} K\left(\sigma_{b y p}, \omega_{\min }, \omega, \tau\right) \frac{\partial}{\partial \tau} C(t, \tau)\right]+\right. \\
& \gamma_{x y}=\frac{2(1+v)}{E_{m}^{0}(t)}\left(1-\mu_{s x y}\right) \tau_{b x y m}(t) S_{m}\left[\tau_{b x y m}(\tau)\left[1+\alpha \mu_{s x y}\right]-2(1+v)\left(1-\mu_{s x y}\right) \tau_{b x y p}(t) S_{p}\left[\tau_{b x y p}(t)\right] .\right. \\
& \cdot\left[1+\mu_{s x y} E_{s} K\left(\tau_{b x y p} \omega_{\min }, \omega, t\right)\right] C\left(t, t_{0}\right)- \\
& -2(1+v)\left(1-\mu_{s x y}\right) \int_{t_{0}}^{t} \tau_{b x y p}(\tau) S_{p}\left[\tau_{b x y p}(\tau)\right]\left[1+\mu_{s x y} E_{s} K\left(\tau_{b x y p}, \omega_{\min }, \omega, \tau\right) \frac{\partial}{\partial \tau} C(t, \tau)\right] d \tau
\end{aligned}
$$

Note that if the dimensions of the elementary volume viewed in different direction greater than at least 5 diameters of the rebar size factor $(1-\mu \mathrm{si})$ can be neglected.

Output stress of one component beyond the surface strength, as noted above, will mean that the corresponding axis direction is formed crack. As is known, the actual tension in the areas where cracks are formed unstable. In fracture zone, all the force is perceived reinforcement, reaching its maximum value, and in the areas between the cracks, they are reduced in view of the strength of adhesion to concrete reinforcement, which in those areas involved in the work (Fig.2). Note that if an elementary volume of the body inclined crack is formed at an angle of more than $30^{\circ}$, agree to consider that this crack will cross not one, but two planes.

In the case of crack along one of the coordinate axis directions, in determining the stress on the faces of the elementary volume, with cracks running, you can use a known prerequisite:

$$
\sigma_{s m i}=\psi_{s i} \mu_{s i} \sigma_{s i} ; \quad \varepsilon_{s m i}=\sigma_{s m i} / E_{s i}=\psi_{s i} \mu_{s i} \sigma_{s i} / E_{s i}
$$

where: $\sigma_{\text {smi }}$ - the average stress in the reinforcement in the area between the cracks; $\psi_{\text {si }}$ coefficient taking into account the effect of concrete in tension and adhesion to concrete reinforcement; $\mu_{\mathrm{si}}$ - reinforcement ratio in this direction; $\sigma_{\mathrm{si}}-$ stress in reinforcement on the section with cracks; $\varepsilon_{\mathrm{smi}}$ - average relative deformation of the reinforcement in the areas between the cracks; $E_{s}-$ modulus of elasticity of reinforcement; $i=x, y, z$.

Said hypothesis can be extended to the case of a triaxial strain state taking into account the non-linearity and rheology, then: 


$$
\begin{aligned}
& \varepsilon_{x}\left(t, t_{0}\right)=\frac{\psi_{s x} \mu_{s x} \sigma_{s x}(t) S_{m}\left[\frac{\sigma_{s x}(t)}{R_{s}^{*}(\rho, t)}\right]-v_{s}\left\{\begin{array}{l}
\psi_{s y} \mu_{s y} \sigma_{s y}(t) S_{m}\left[\frac{\sigma_{s y}(t)}{R_{s}^{*}(\rho, t)}\right]+ \\
+\psi_{s z} \mu_{s z} \sigma_{s z}(t) S_{m}\left[\frac{\sigma_{s z}(t)}{R_{s}^{*}(\rho, t)}\right]
\end{array}\right\}}{E_{s}^{0}(t)}+ \\
& +\left\{\psi_{s x} \mu_{s x} \sigma_{s x}(t) S_{p}\left[\frac{\sigma_{s x}(t)}{R_{s}^{*}(\rho, t)}\right] K\left(\sigma_{s x}, \omega_{\min }, \omega, t\right)-v\left(\begin{array}{l}
\psi_{s y} \mu_{s y} \sigma_{s y}(t) S_{p}\left[\frac{\sigma_{s y}(t)}{R_{s}^{*}(\rho, t)}\right] K_{s}\left(\sigma_{s y}, \omega_{\min }, \omega, t\right)+ \\
+\psi_{s z} \sigma_{s z}(t) S_{p}\left[\frac{\sigma_{s z}(t)}{R_{s}^{*}(\rho, t)}\right] K\left(\sigma_{s z}, \omega_{\min }, \omega, t\right)
\end{array}\right)\right\} C_{s}\left(t, t_{0}\right)- \\
& -\int_{t_{0}}^{t} \psi_{s x} \mu_{s x} \sigma_{s x}(\tau) S_{p}\left[\frac{\sigma_{s x}(\tau)}{R_{s}^{*}(\rho, \tau)}\right] K\left(\sigma_{s x}, \omega_{\min }, \omega, \tau\right) \frac{\partial}{\partial \tau} C_{s}(\tau, t) d \tau+ \\
& +v_{s} \int_{t_{0}}^{t} \psi_{s y} \mu_{s y} \sigma_{s y}(\tau) S_{p}\left[\frac{\sigma_{s y}(\tau)}{R_{s}^{*}(\rho, \tau)}\right] K\left(\sigma_{s y}, \omega_{\min }, \omega, \tau\right) \frac{\partial}{\partial \tau} C_{s}(\tau, t) d \tau+ \\
& +v_{s} \int_{t_{0}}^{t} \psi_{s z} \mu_{s z} \sigma_{s z}(\tau) S_{p}\left[\frac{\sigma_{s z}(\tau)}{R_{s}^{*}(\rho, \tau)}\right] K\left(\sigma_{s z}, \omega_{\min }, \omega, \tau\right) \frac{\partial}{\partial \tau} C_{s}(\tau, t) d \tau
\end{aligned}
$$

here: $v_{\mathrm{s}}$ - coefficient of relative transverse strain of reinforcing steel. The value $\psi_{\mathrm{s}}$ coefficient with sufficient accuracy to determine the permissible from the known empirical formula [4], or other well-known relationship:

$$
\psi_{s i}=1,25-\varphi_{l s} \frac{R^{*}{ }_{b t}(\rho)}{\sigma_{s i} S_{s}\left(\sigma_{s i}\right)}
$$

where: $\varphi_{\mathrm{ls}}$ - empirical coefficient depending on the duration of action of an external load and concrete class; $\mathrm{S}_{\mathrm{s}}\left(\sigma_{\mathrm{s}}\right)$ - nonlinearity function of reinforcement deformation. Index $\mathrm{s}$ in formulas (28) and (29) means that it is, on deformation of the reinforcing steel. In accordance with the requirements of the rules on the calculation of the dynamic impact factor the value $\psi_{\text {s }}$ encouraged to take equal to 1 , due to the loss of adhesion between reinforcement and concrete during the vibrations, it is permissible for the smooth reinforcement, but hardly true for reinforcement of periodic profile.

Possible to use the method proposed in [4-8] for the tangential stresses, where their value depends on the tangential displacements of reinforcement and a crack is determined by theoretical and experimental data. In these dependencies the magnitude of compliance of rods under tangential displacements depends on concrete bases give collapse under the reinforcement (posted by using the so-called coefficient dowel effect):

$$
\begin{aligned}
& \gamma_{x y}=\frac{2\left(1+v_{s}\right)}{E_{s}^{0}(t)} \psi_{s x} n_{x x} \mu_{s y y} \tau_{s y y}(t) S_{m}\left[\frac{\tau_{s y}(t)}{R_{r}^{*}(\rho, t)}\right]+2(1+v) \psi_{s x} n_{x x} \tau_{s x y} S_{m}\left[\frac{\tau_{s y y}(t)}{R_{r}^{*}(\rho, t)}\right] K\left(\tau_{s x y}, \omega_{\min }, \omega, y\right) . \\
& \cdot C_{s}\left(t, t_{0}\right)-2(1+v) \int_{t_{0}}^{t} \psi_{s x} n_{x} \mu_{s x y} \tau_{s x y}(\tau) S_{p}\left[\frac{\tau_{s y}(\tau)}{R_{r}^{*}(\rho, \tau)}\right] K\left(\tau_{s x y}, \omega_{\min }, \omega, \tau\right) \frac{\partial}{\partial \tau} C_{s}(t, \tau) d \tau
\end{aligned}
$$

$\tau_{\mathrm{sij}}$ - shear stresses along the respective axes; $\mathrm{E}_{\mathrm{s}}$ and $\psi_{\mathrm{si}}$ - have the same meanings as in (27); $\mu_{\mathrm{sij}}$ - are determined by the expressions (24); $\mathrm{n}_{\tau i}$ - factor dowel effect, defined by the formula:

$$
\mathrm{n}_{\tau \mathrm{i}}=0,75 \mathrm{E}_{\mathrm{s}} \eta / \mathrm{E}_{\mathrm{m}}^{0}(\mathrm{t})
$$


here: $\eta$ - the ratio of stresses equal to:

$$
\eta=\sigma_{\text {ni }} / \sigma_{\text {ncrc }}>=1
$$

$\sigma_{n i}-(i=x, y, z)$ - effective stress along the corresponding coordinate axis; $\sigma_{\text {ncrc }}$ - concrete stress along the longitudinal axis at the moment of fracture.

It should be noted that according to (30) are valid only in the case of equity law pairing shear stresses that rigorous formulation does not correspond to reality, since the deformation of concrete with cracks more in line with the conditions of deformation of the orthotropic material, especially if in one direction the crack appeared, and the other not. However, due to the fact that the difference in shear stresses have little effect on the stress strain state of the unit cell of the finite element method, we agree to assume with some approximation of shares that the law of pairing shear stresses fair. This assumption will greatly simplify the proposed methodology of calculation.

Note that in some cases, for certain types of reinforcement is allowed, with some degree of accuracy, linearity, and ignore the rheology deformation, it becomes possible by using high-strength and heat-hardened steels at low levels of operating stresses. Then deformations in the presence of cracks will form:

$$
\begin{gathered}
\varepsilon_{x}=\frac{1}{E_{s}}\left[\psi_{s x} \mu_{s x} \sigma_{s x}-v_{s}\left(\psi_{s y} \mu_{s y} \sigma_{s y}+\psi_{s z} \mu_{s z} \sigma_{s z}\right)\right] \\
\varepsilon_{y}=\frac{1}{E_{s}}\left[\psi_{s y} \mu_{s y} \sigma_{s y}-v_{s}\left(\psi_{s x} \mu_{s x} \sigma_{s x}+\psi_{s z} \mu_{s z} \sigma_{s z}\right)\right] \\
\varepsilon_{z}=\frac{1}{E_{s}}\left[\psi_{s z} \mu_{s z} \sigma_{s z}-v_{s}\left(\psi_{s x} \mu_{s x} \sigma_{s x}+\psi_{s y} \mu_{s y} \sigma_{s y}\right)\right] \\
\gamma_{x y}=\frac{2\left(1+v_{s}\right)}{E_{s}^{0}} \psi_{s x} n_{\tau x} \mu_{s y y} \tau_{s x y} \quad \gamma_{y z}=\frac{2\left(1+v_{s}\right)}{E_{s}^{0}} \psi_{s y} n_{\tau y} \mu_{s y z} \tau_{s y z} ; \quad \gamma_{z x}=\frac{2\left(1+v_{s}\right)}{E_{s}^{0}} \psi_{s z} n_{t z} \mu_{s z x} \tau_{s z x}
\end{gathered}
$$

Thus, according to (28), (30), (33) and (34) allow us to find the values of the components of axial and shear strain under triaxial stress-strain state after the formation of cracks. In the absence of cracks, or along a direction corresponding to the calculation of stresses or deformation of the components to be determined using relations (15) and (16), and in the absence of reinforcement at - (19) or (22). The value of the width of the cracks is allowed to determine the known conventional empirical method [2], with the only difference being that in the calculation of stresses in the reinforcing rods should consider the impact of power imperfections deformation of materials. Allowance in the calculation vibrocreep reinforcement factor by using formulas (18), (23) and (27) for calculating values of stresses. However, rheological parameters may be neglected, since the creep does not have time to appear during one oscillation cycle.

\section{Conclusions}

Summarizing the above, we can say that the effect of reinforcement on the work of concrete in three-dimensional stressed state is considered, and also the basic phenomenological resolving equations are developed under conditions of a triaxial stress-strain state under vibrational influences for the calculation of reinforced concrete elements working with or without cracks in conditions of nonlinearity and rheology of deformation. It should be noted that the use of the proposed calculation technique will lead to significant mathematical difficulties. 
Technique of mathematical calculations in the general case, both in the presence or absence of the reinforcement and cracks in different directions detail in the future, in the development of algorithms for dynamic analysis of reinforced concrete structures and foundations in conditions of triaxial stress-strain state. Progress in solving the problem and the sequence of mathematical dependencies are assigned to the implementation of the mathematical algorithm of integrated assessment method, and depend ultimately on the level of the stress state in each cell of the finite-element mesh, which determines the operation of the material to cracking or after their formation. Such complexity of the mathematical apparatus can be successfully overcome and obtain the desired result with the help of linearization based on the method of integral estimates, including the fixation of the creep processes in the varied stresses at the material time of observation, as well as the use iterative processes in the implementation of the finite element method for solving engineering problems.

\section{References}

1. V.M. Bondarenko, V.I. Rimshin The theory of dissipative power resistance of reinforced concrete, 287 (2015)

2. E.M. Zverjaev, M. V. Berlinov, M. N. Berlinova. International Journal of Applied Engineering Research, 11(8), 5811 (2016)

3. E.A. Korol, M. V. Berlinov, M.N. Berlinova, Procedia Engineering, 165, 292 (2016)

4. E.A. Korol, Yu.A. Kharkin, Book of Reports of XX Russian-Polish-Slovak Workshop "Theoretical Fundamentals of Construction" 401 (2011)

5. V.M. Bondarenko,V.I.Kolchunov. Calculation models of the power resistance of reinforced concrete, 112 (2004)

6. P. Petronijević, N. Ivanišević, M. Rakočević, D. Arizanović, Journal of Applied Engineering Science 1(10), 43-48 (2012) ISSN 1451-4117, doi:10.5937/jaes10-1664

7. M. Lazarevska, M. Knežević, M. Cvetkovska, N. Ivanišević, T. Samardžioska, A. Trombeva-Gavriloska, Gradjevinar 7/2012(64), 565-571 (2012) ISSN (on line): 13339095; ISSN (printed) 0350-2465

8. M. Rakočević, S. Popović, N. Ivanišević, Composites Part B 122, 202-218 (2017) doi: 10.1016/ j.compositesb.2017.03.044 